\title{
Series of primitive right-angled triangles
}

\author{
Martin W. Bredenkamp \\ Department of Science, Asia-Pacific International University; PO Box 4, MuakLek, Saraburi Province, 18180, Thailand
}

Email address:

martinb@apiu.edu (M. W. Bredenkamp)

To cite this article:

Martin W. Bredenkamp. Series of Primitive Right-Angled Triangles. Applied and Computational Mathematics. Vol. 2, No. 2, 2013, pp. 42-53. doi: 10.11648/j.acm.20130202.15

\begin{abstract}
From the infinite matrix of right-angled triangles, series of triangles are found that approach a right-angled triangle that has one irrational side such as the $45^{\circ}$ triangle. This allows for the creation of a series of fractions that have as their limit an irrational number. Formulae for finding the next triangle in the triangle series, and thus the next fraction in the fraction series, are also developed. Such a series can be found for the square root of every uneven number that is not a perfect square, and for those of some of the even numbers as well.
\end{abstract}

Keywords: Pythagorean Triple Series, Non-Pythagorean Right-Angled Triangle Limit, Rational Series With Irrational Limits

\section{Introduction}

Having established defining indices that position all primitive right-angled triangles in an infinite two-dimensional matrix [1], which may be defined as the universal set, we have gone about finding subsets of right-angled triangles that are very similar in proportion. The obvious place to start is to find triangles that approximate the $45^{\circ}$ triangle, and the $30 / 60^{\circ}$ triangle that are the standard set squares. This quest has led to finding and defining series of primitive right-angled triangles that in proportion converge to the said triangles which have irrational proportions between all but one pair of sides. This also gives rise to series of fractions that converge to an irrational number.

\section{Approximations to the $45^{\circ}$ Triangle}

In the previous paper [1] it has been established that all primitive right-angled triangles are comprised of an uneven-numbered leg $(u)$, an even-numbered leg $(e)$ and the hypotenuse $(h)$ which in itself is uneven-numbered (all variables are positive integers). This eliminates the possibility of having the $45^{\circ}$ triangle as part of the subset of approximating primitive right-angled triangles. This is well known in any case, since the hypotenuse is $\sqrt{2}$, which is an irrational number, disqualifying it from the set of primitive right-angled triangles.

Let us define the subset of triangles, and then set about finding them. Let us define them in terms of the indices $(i, j)$ developed in the previous paper [1]. We define the subset of triangles (A) as follows:

$$
\begin{gathered}
\mathrm{A}=\left\{(i, j) \mid u=i^{2}+i j, e=j^{2} / 2+i j, h^{2}=u^{2}+e^{2}, \text { and }|e-u|=1,\right. \\
\text { where }(i+1) / 2 \text { and } j / 2 \in \mathbb{N}\}
\end{gathered}
$$

In the definition of the subset of triangles (A), an arbitrary value of 1 was chosen as the limit to the difference between the legs of the triangle. It does not take long to realise that such triangles exist, since the most well known primitive right-angled triangle, the $3 / 4 / 5$-triangle $[(i, j)=$ $(1,2)]$, is one where $4-3=1$. A closer look at the matrix of triangles shows that finding others is not such a daunting task, since for every $i, u_{j=2-\infty}$ is an arithmetic sequence with the interval being equal to $2 i$. On the other hand, $e_{j=2-\infty}$ is a series with quadratic properties, since the interval in the series is defined by $\left[(j+2)^{2}-j^{2}\right] / 2+2 i$, which increases as $j$ increases. Since for all cases except where $i=1$, the series begins with $e$ smaller than $u$, and since the intervals for $e$ in a series are increasing quadratically, $e$ will pass the value of $u$ somewhere in the series. The task is therefore to find the place in each series where $e$ passes $u$, enabling the easy identification of a case where $|e-u|=1$.

The $3 / 4 / 5$-triangle $[(i, j)=(1,2)]$ belongs to the first series $(i=1)$. The second series, where $i=3$, also has such a triangle: $21 / 20 / 29$-triangle $[(i, j)=(3,4)]$. The third series $(i=$ 5) does not have such a triangle: for $(i, j)=(5,6), e(48)$ is less than $u(55)$, by 7 ; and for $(i, j)=(5,8), e(72)$ is greater than $u(65)$ by 7 . The fourth series $(i=7)$, in turn, has such a triangle again: $119 / 120 / 169$-triangle $[(i, j)=(7,10)]$. The next series to have a qualifying triangle is the ninth $(i=17)$ : $697 / 696 / 985$-triangle $[(i, j)=(17,24)]$. We thus see that this 
subset of primitve right-angled triangles exists.

This subset of triangles may be ordered according to the numerical values of $(i, j)$, forming a series of triangles. The size of the triangles increases in the series. Since the series is defined as having the legs $u$ and $e$, differing by 1 only, the difference becomes less significant as the triangles increase in size. This means that the legs are more and more equal as the series progresses, and the triangles resemble the $45^{\circ}$ triangle more and more. This also means that the ratio between $h$ and $u$ or $e$ better and better approximates $\sqrt{2}$ as the series progresses.

As the series progresses, the interval from $i$ to the next $i$, containing such a triangle also increases, making it more and more difficult to systematically and manually find the next member in the series. The question then arises: Is there a mathematical equation that defines this series of triangles, so that they may be determined algebraically? Having systematically found the first few triangles in the series, an investigation of $(i, j)$ reveals an algebraic pattern:

For the series $(i, j)_{n},(i, j)_{n+1}=\left(i_{n}+j_{n}, 2 i_{n}+j_{n}\right)$

Table 1 contains the first fifteen triangles in this series, the latter part having been calculated by the above formula. Also included is $\sqrt{2}$, and the calculated approximation to $\sqrt{2}$, offered by each respective triangle. As the series progresses, the approximation improves, as indicated by the underlined digits that coincide with $\sqrt{2}$. The ratio of $j / i$ is also included to show that it yields a series that also approximates a number, which happens to also be $\sqrt{2}$, but for another reason, which will come to light later in this paper.

Table 1. The series of triangles $(i, j)_{n}$ that have as their limit the $45^{\circ}$ triangle.

\begin{tabular}{|c|c|c|c|c|c|c|c|}
\hline$n$ & $i$ & $i$ & $u$ & $e$ & $\boldsymbol{h}$ & $\begin{array}{l}h / e(\sqrt{2}) \\
1.4142135623730950\end{array}$ & $j / i$ \\
\hline 1 & 1 & 2 & 3 & 4 & 5 & 1.25 & 2 \\
\hline 2 & 3 & 4 & 21 & 20 & 29 & $\underline{1.45}$ & $\underline{1.333333333333}$ \\
\hline 3 & 7 & 10 & 119 & 120 & 169 & $\underline{1.4083333333333333}$ & $\underline{1.428571428571}$ \\
\hline 4 & 17 & 24 & 697 & 696 & 985 & $\underline{1.4152298850574713}$ & $\underline{1.41} 1764705882$ \\
\hline 5 & 41 & 58 & 4059 & 4060 & 5741 & 1.4140394088669951 & 1.414634146341 \\
\hline 6 & 99 & 140 & 23661 & 23660 & 33461 & 1.4142434488588335 & $\underline{1.414141414141}$ \\
\hline 7 & 239 & 338 & 137903 & 137904 & 195025 & $\underline{1.4142084348532312}$ & $\underline{1.414225941423}$ \\
\hline 8 & 577 & 816 & 803761 & 803760 & 1136689 & $\underline{1.4142144421220265}$ & $\underline{1.414211438475}$ \\
\hline 9 & 1393 & 1970 & 4684659 & 4684660 & 6625109 & $\underline{1.4142134114322064}$ & $\underline{1.414213} 926777$ \\
\hline 10 & 3363 & 4756 & 27304197 & 27304196 & 38613965 & $\underline{1.4142135882704622}$ & $\underline{1.414213499851}$ \\
\hline 11 & 8119 & 11482 & 159140519 & 159140520 & 225058681 & 1.4142135579298095 & $\underline{1.414213573100}$ \\
\hline 12 & 19601 & 27720 & 927538921 & 927538920 & 1311738121 & $\underline{1.4142135631354423}$ & $\underline{1.414213560533}$ \\
\hline 13 & 47321 & 66922 & 5406093003 & 5406093004 & 7645370045 & $\underline{1.4142135622422969}$ & $\underline{1.414213562689}$ \\
\hline 14 & 114243 & 161564 & 31509019101 & 31509019100 & 44560482149 & $\underline{1.4142135623} 955365$ & $\underline{1.414213562319}$ \\
\hline 15 & 275807 & 390050 & 183648021599 & 183648021600 & 259717522849 & 1.4142135623692447 & $\underline{1.414213562382}$ \\
\hline
\end{tabular}

An interesting observation is that when $n$ is uneven, $u$ is less than $e$ by 1 , and when $n$ is even, $u$ is greater than $e$ by 1 . Another observation is that a similar series of rational numbers leading to $\sqrt{2}$ is $h / u$.
Having defined $(i, j)_{n+1}$ in terms of $i_{n}$ and $j_{n}$, we can now define the sides of the triangle $(u, e, h)_{n+1}$ in terms of $i_{n}$ and $j_{n}$, $\left[(2),(6)\right.$ and (8) respectively] and then in terms of $u_{n}, e_{n}$ and $h_{n}[(5),(7)$ and (9) respectively]. This calls for a good exercise in algebra, which sometimes includes factorisation:

$$
\begin{aligned}
u_{n+1} & =i_{n+1}^{2}+i_{n+1} j_{n+1} \\
& =\left(i_{n}+j_{n}\right)^{2}+\left(i_{n}+j_{n}\right)\left(2 i_{n}+j_{n}\right) \\
& =i_{n}^{2}+2 i_{n} j_{n}+j_{n}^{2}+2 i_{n}^{2}+3 i_{n} j_{n}+j_{n}^{2} \\
& =3 i_{n}^{2}+5 i_{n} j_{n}+2 j_{n}^{2}
\end{aligned}
$$

by definition [1]

$$
\begin{aligned}
& \left(u=i^{2}+i j\right) \\
& {\left[(i, j)_{n+1}=\left(i_{n}+j_{n}, 2 i_{n}+j_{n}\right)\right]}
\end{aligned}
$$$$
\therefore i=\sqrt{h-e}
$$

However $h=i^{2}+e$ 


$$
\begin{array}{rlrl}
\text { and } e & =i j+j^{2} / 2 & & \text { by definition (see also ref [1]) } \\
\therefore 2 e & =2 j \sqrt{h-e}+j^{2} & \\
\therefore 0 & =j^{2}+2 j \sqrt{h-e}-2 e & \\
\therefore & =\frac{-2 \sqrt{h-e}+\sqrt{4(h-e)+8 e}}{2} & & \text { roots of a quadratic equation } \\
& =\frac{-2 \sqrt{h-e}+2 \sqrt{h-e+2 e}}{2} & \\
& =\sqrt{h+e}-\sqrt{h-e} & \\
\therefore u_{n+1} & =3\left(\sqrt{h_{n}-e_{n}}\right)^{2}+5 \sqrt{h_{n}-e_{n}} \cdot\left(\sqrt{h_{n}+e_{n}}-\sqrt{h_{n}-e_{n}}\right)+2\left(\sqrt{h_{n}+e_{n}}-\sqrt{h_{n}-e_{n}}\right)^{2}
\end{array}
$$$$
=3 h_{n}-3 e_{n}+5\left(\sqrt{h_{n}^{2}-e_{n}^{2}}-h_{n}+e_{n}\right)+2\left(h_{n}+e_{n}-2 \sqrt{h_{n}^{2}-e_{n}^{2}}+h_{n}-e_{n}\right)
$$$$
=3 h_{n}-3 e_{n}+5\left(u_{n}-h_{n}+e_{n}\right)+2\left(2 h_{\mathrm{n}}-2 u_{n}\right) \quad \text { by Pythagorus }
$$$$
=2 h_{n}+2 e_{n}+u_{n}
$$$$
e_{n+1}=j_{n+1}^{2} / 2+i_{n+1} j_{n+1}
$$$$
\therefore 2 e_{n+1}=\left(2 i_{n}+j_{n}\right)^{2}+2\left(i_{n}+j_{n}\right)\left(2 i_{n}+j_{n}\right)
$$$$
=4 i_{n}^{2}+4 i_{n} j_{n}+j_{n}^{2}+4 i_{n}^{2}+6 i_{n} j_{n}+2 j_{n}^{2}
$$$$
=8 i_{n}^{2}+10 i_{n} j_{n}+3 j_{n}^{2}
$$$$
=\left(4 i_{n}+3 j_{n}\right)\left(2 i_{n}+j_{n}\right)
$$$$
=\left[4 \sqrt{h_{n}-e_{n}}+3\left(\sqrt{h_{n}+e_{n}}-\sqrt{h_{n}-e_{n}}\right)\right]\left(2 \sqrt{h_{n}-e_{n}}+\sqrt{h_{n}+e_{n}}-\sqrt{h_{n}-e_{n}}\right)
$$$$
\left(e=j^{2} / 2+i j\right)
$$$$
\left[(i, j)_{n+1}=\left(i_{n}+j_{n}, 2 i_{n}+j_{n}\right)\right](1)
$$$$
=\left(\sqrt{h_{n}-e_{n}}+3 \sqrt{h_{n}+e_{n}}\right)\left(\sqrt{h_{n}-e_{n}}+\sqrt{h_{n}+e_{n}}\right)
$$$$
=h_{n}-e_{n}+4 u_{n}+3 h_{n}+3 e_{n}
$$$$
\text { by Pythagorus and diff. of squares }
$$$$
=4 h_{n}+2 e_{n}+4 u_{n}
$$$$
\therefore e_{n+1}=2 h_{n}+e_{n}+2 u_{n}
$$$$
h_{n+1}=i_{n+1}^{2}+i_{n+1} j_{n+1}+j_{n+1}^{2} / 2
$$$$
\left(h=i^{2}+i j+j^{2} / 2\right)
$$$$
=e_{n+1}+i_{n+1}^{2}
$$$$
\left(e=j^{2} / 2+i j\right)
$$$$
2 h_{n+1}=8 i_{n}^{2}+10 i_{n} j_{n}+3 j_{n}^{2}+2 i_{n}^{2}+4 i_{n} j_{n}+2 j_{n}^{2}
$$$$
\left[(i, j)_{n+1}=\left(i_{n}+j_{n}, 2 i_{n}+j_{n}\right)\right](1) \text { and }(6)
$$$$
=10 i_{n}^{2}+14 i_{n} j_{n}+5 j_{n}^{2}
$$$$
=10\left(\sqrt{h_{n}-e_{n}}\right)^{2}+14 \sqrt{h_{n}-e_{n}} \cdot\left(\sqrt{h_{n}+e_{n}}-\sqrt{h_{n}-e_{n}}\right)+5\left(\sqrt{h_{n}+e_{n}}-\sqrt{h_{n}-e_{n}}\right)^{2}
$$

$$
=10 h_{n}-10 e_{n}+14\left(\sqrt{h_{n}^{2}-e_{n}^{2}}-h_{n}+e_{n}\right)+5\left(h_{n}+e_{n}-2 \sqrt{h_{n}^{2}-e_{n}^{2}}+h_{n}-e_{n}\right)
$$$$
=10 h_{n}-10 e_{n}+14\left(u_{n}-h_{n}+e_{n}\right)+5\left(2 h_{n}-2 u_{n}\right) \quad \text { by Pythagorus }
$$$$
=6 h_{n}+4 e_{n}+4 u_{n}
$$$$
\therefore h_{n+1}=3 h_{n}+2 e_{n}+2 u_{n}
$$

It takes more algebra to solve for $h$ in terms of $h$ only, etc It is especially complicated with the even leg being greater than the uneven leg for the uneven positional-numbered members of the series, and the even leg being less than the uneven leg for the even positional-numbered members of the series.

$$
h_{n+1}=6 h_{n}-h_{n-1}
$$

For the next even positional-numbered member of the series $(n+1$ as even): 


$$
\begin{array}{r}
e_{n+1}=6 e_{\mathrm{n}}-e_{n-1}-4 \\
\text { and } u_{\mathrm{n}+1}=6 u_{n}-u_{n-1}+4
\end{array}
$$

And for the next uneven positional-numbered members of the series $(n+1$ as uneven):

$$
\begin{array}{r}
e_{n+1}=6 e_{\mathrm{n}}-e_{n-1}+4 \\
\text { and } u_{\mathrm{n}+1}=6 u_{n}-u_{n-1}-4
\end{array}
$$

Since both the $e$ and the $u$ legs may be used for calculat- ing $\sqrt{2}$, the legs may be dealt with as the greater number and the lesser number, irrespective of whether they are even or uneven. This simplifies the formulae to one each for each leg. Let the lesser leg be $l$, and the greater leg $g$, then:

$$
\begin{aligned}
g_{n+1} & =6 g_{n}-g_{n-1}-2 \\
\text { and } l_{n+1} & =6 l_{n}-l_{n-1}+2
\end{aligned}
$$

Now, the simple formulae for a series of rational numbers that have the square $\sqrt{2}$ as their limit are the following:

$$
\begin{array}{ll}
\hline \text { For the greater numbers: } \frac{h_{n+1}}{g_{n+1}} & \text { where } h_{1}=5, h_{2}=29, h_{n+1}=6 h_{n}-h_{n-1}, \\
& \text { And } g_{1}=4, g_{2}=21, g_{n+1}=6 g_{n}-g_{n-1}-2 . \\
\text { For the lesser numbers: } \frac{h_{n+1}}{l_{n+1}} & \text { where } h_{1}=5, h_{2}=29, h_{n+1}=6 h_{n}-h_{n-1}, \\
& \text { and } l_{1}=3, l_{2}=20, l_{n+1}=6 l_{n}-l_{n-1}+2 .
\end{array}
$$

\section{Approximations to the $60^{\circ}$ Triangle}

The $60^{\circ}$ triangle is one half of a bisected equilateral triangle with sides of 2 units. The hypotenuse, therefore, is 2 units long, and the short leg is 1 unit long. The leg of bisection is, by Pythagorus' theorem, the root of the difference of the squares of 2 and 1 ie $\sqrt{3}$. As with the $45^{\circ}$ triangle, we will not only develop a series of triangles that approximate the $60^{\circ}$ triangle more and more, but we will also develop a series of rational numbers that will have $\sqrt{3}$ as their limit.

We can define the subset of triangles (B), where the even leg $(e)$ is the bisected leg, and the uneven leg $(u)$ the short leg, as follows:

$$
\begin{gathered}
\mathrm{B}=\left\{(i, j) \mid u=i^{2}+i j, e=j^{2} / 2+i j, h^{2}=u^{2}+e^{2},\right. \\
\text { and }|h-2 u|=1, \text { where }(i+1) / 2 \text { and } j / 2 \in \mathbb{N}\}
\end{gathered}
$$

Let us call this series of triangles the $\boldsymbol{e}$-series of $\sqrt{\mathbf{3}}$ since $e$ represents the irrational leg in a system where the series are defined by their irrational legs. To qualify for this subset of triangles (B), a triangle must have its hypotenuse within 1 of two times the uneven leg. Again, the 3/4/5- triangle $[(i, j)=(1,2)]$ qualifies to be an element of $\mathrm{B}$, where $|5-2(3)|=1$. As before, for every series $i$, twice the uneven leg $(u)$ starts off greater than $h$, but the latter increases quadratically, versus arithmetically, and a cross-over point is reached, where the presence of a qualifying triangle is sought for. The second series $(i=3)$ contains the second triangle: $33 / 56 / 65$-triangle $[(i, j)=(3,8)] ;$ and the sixth series $(i=11)$ the third triangle: $451 / 780 / 901$-triangle $[(i, j)$ $=(11,30)]$, etc. The mathematical equation to define each member of this series is:

$$
\text { For the series }(i, j)_{\mathrm{n}},(i, j)_{n+1}=\left(i_{n}+j_{n}, 2 i_{n}+3 j_{n}\right)
$$

\begin{tabular}{|c|c|c|c|c|c|c|c|}
\hline & $i$ & $i$ & $u$ & $e$ & $h$ & $\begin{array}{l}e / u(\sqrt{3}) \\
1.7320508075688773\end{array}$ & $i / i$ \\
\hline 1 & 1 & 2 & 3 & 4 & 5 & 1.33333333333333333 & 2 \\
\hline 2 & 3 & 8 & 33 & 56 & 65 & $\underline{1} .6969696969696970$ & 2.666666666667 \\
\hline 3 & 11 & 30 & 451 & 780 & 901 & 1.7294900221729490 & 2.727272727273 \\
\hline 4 & 41 & 112 & 6273 & 10864 & 12545 & 1.7318667304320102 & $2 . \overline{73} 1707317073$ \\
\hline 5 & 153 & 418 & 87363 & 151316 & 174725 & 1.7320375902842164 & 2.732026143791 \\
\hline 6 & 571 & 1560 & 1216801 & 2107560 & 2433601 & 1.7320498586046527 & 2.732049036778 \\
\hline 7 & 2131 & 5822 & 16947843 & 29354524 & 33895685 & 1.7320507394362811 & 2.732050680432 \\
\hline 8 & 7953 & 21728 & 236052993 & 408855776 & 472105985 & 1.7320508026771768 & $2 . \overline{732050} 798441$ \\
\hline 9 & 29681 & 81090 & 3287794051 & 5694626340 & 6575588101 & 1.7320508072176690 & 2.732050806914 \\
\hline 10 & 110771 & 302632 & 45793063713 & 79315912984 & 91586127425 & 1.7320508075436616 & $2 . \overline{732050807522}$ \\
\hline 11 & 413403 & 1129438 & 637815097923 & 1104728155436 & 1275630195845 & 1.7320508075670668 & 2.732050807565 \\
\hline
\end{tabular}

Table 2 contains the first eleven triangles in this series. Also included is $\sqrt{3}$, and the calculated approximation to $\sqrt{3}(e / u)$, offered by each respective triangle. Again the ratio of $j / i$ is included to show that it yields a series that also approximates a number that will have meaning later in this section. Take note that the decimal portion of $j / i$ is equal to the decimal portion of $\sqrt{3}$. The progressive underlined decimal part of each number shows to what extent it agrees with the decimal portion of $\sqrt{3}$.

Table 2. The series of triangles $(i, j)_{n}$ that have as their limit the $60^{\circ}$ triangle. 
In this series of triangles, $2 u$ is always 1 greater than $h$.

As before the sides of the triangles $(u, e, h)_{n+1}$ may be defined in terms of $i_{n}$ and $j_{n}$, [(20), (22) and (24) respectively] and in terms of $u_{n}, e_{n}$ and $h_{n}$ [(21), (23) and (25) respectively]. The algebra leading up to the equations is not included:

$$
\begin{array}{ll}
u_{n+1} & =3 i_{n}^{2}+7 i_{n} j_{n}+4 j_{n}^{2} \\
& =4 h_{n}+4 e_{n}-u_{n} \\
2 e_{n+1} & =\left(2 i_{n}+3 j_{n}\right)\left(4 i_{n}+5 j_{n}\right) \\
\text { and } e_{n+1} & =8 h_{n}+7 e_{n}-4 u_{n} \\
2 h_{n+1} & =10 i_{n}^{2}+26 i_{n} j_{n}+19 j_{n}^{2} \\
\text { and } h_{n+1} & =9 h_{n}+8 e_{n}-4 u_{n}
\end{array}
$$

Once again $h$ may be solved in terms of $h$ only, etc. In this series there is no alternation, so the formulae are simple:

$$
\begin{aligned}
h_{n+1} & =15 h_{n}-15 h_{n-1}+h_{n-2} \\
e_{n+1} & =15 e_{n}-15 e_{n-1}+e_{n-2} \\
\text { and } u_{n+1} & =15 u_{n}-15 u_{n-1}+u_{n-2}
\end{aligned}
$$

Therefore the simple formula for a series of rational numbers that has $\sqrt{3}$ as its limit is the following:

$$
\begin{aligned}
\frac{e_{n+1}}{u_{n+1}} \text { where } & e_{1}=4, e_{2}=56, e_{3}=780, \\
& e_{n+1}=15 e_{n}-15 e_{n-1}+e_{n-2}, \\
\text { and } & u_{1}=3, u_{2}=33, u_{3}=451, \\
& u_{n+1}=15 u_{n}-15 u_{n-1}+u_{n-2}
\end{aligned}
$$

In the beginning of this section we arbitrarily chose the even leg $(e)$ to represent the $\sqrt{3}-\mathrm{leg}$ of the $60^{\circ}$ triangle forming the $e$-series of $\sqrt{3}$. Now, let the even leg $(e)$ represent the short leg, and the uneven leg $(u)$ represent the bisected leg, defining the subset of triangles (C) as follows:

$$
\begin{gathered}
\mathrm{C}=\left\{(i, j) \mid u=i^{2}+i j, e=j^{2} / 2+\mathrm{ij}, h^{2}=u^{2}+e^{2},\right. \\
\text { and }|h-2 e|=1, \text { where }(i+1) / 2 \text { and } j / 2 \in \mathbb{N}\}
\end{gathered}
$$

This then is the $\boldsymbol{u}$-series of $\sqrt{\mathbf{3}}$ where $u$ represents the irrational leg. Here, the first qualifying triangle belongs to the second series $(i=3)$ : the $15 / 8 / 17$-triangle $[(i, j)=(3,2)]$, where $|17-2(8)|=1$. The second triangle belongs to the sixth series $(i=11)$ : 209/120/241-triangle $[(i, j)=(11,8)]$, etc. The mathematical equation to define each member of this series is:

$$
\text { For the series }(i, j)_{n},(i, j)_{n+1}=\left(3 i_{n}+j_{n}, 2 i_{n}+j_{n}\right)
$$

Table 3 contains the first ten triangles in this series. As before, $\sqrt{3}$ is included, and the calculated approximation to $\sqrt{3}(u / e)$, offered by each respective triangle. The ratio of $j / i$ is also included again.

Table 3. The alternate series of triangles $(i, j)_{n}$ that have as their limit the $60^{\circ}$ triangle.

\begin{tabular}{lllllll}
\hline $\boldsymbol{n}$ & $\boldsymbol{i}$ & $\boldsymbol{i}$ & $\boldsymbol{u}$ & $\boldsymbol{e}$ & $\boldsymbol{h}$ & \multicolumn{1}{c}{$\boldsymbol{u} / \boldsymbol{e}(\sqrt{\mathbf{3}})$} \\
$\mathbf{1 . 7 3 2 0 5 0 8 0 7 5 6 8 8 7 7 3}$
\end{tabular}

The product of the $j / i$ ratios of the $e$-series and the $u$ series is 2, viz. $2.732050807565 \times 0.732050807565=2$. An uncanny property of these two ratios is that the decimal portions of the two ratios are identical, and the integer portion is 2 for the $e$-series, and 0 for the $u$-series. Furthermore, these decimal portions are identical with that of $\sqrt{3}$, which has the intermediate, 1 , as the integer portion of the number. This phenomenon can be justified algebraically. If we break up the ratio of the $e$-series into its integer and decimal components, and set the decimal as an unknown, $x$, to solve where the product of the ratios must be equal to 2 , we have the following:

$$
(2+x) x=2
$$

$$
\begin{gathered}
\therefore 2 x+x^{2}=2 \\
\therefore x^{2}+2 x-2=0 \\
\frac{\sqrt{4+8}}{2} \text { quadratic eq } \\
=-1 \mp \sqrt{3}
\end{gathered}
$$$$
x=\frac{-2 \mp \sqrt{4+8}}{2} \text { quadratic equation }
$$$$
=0.7320508075688773
$$

(rejecting the negative root)

Interestingly the product of the two ratios of the $45^{\circ}$ is also 2, where the legs are the same length and the two series are intertwined $i e \sqrt{2}$ squared is 2 . 
Another interesting phenomenon shows up: The developing decimal portions of the two series are also the same, out of phase by one position in the respective series (see Table 4). This indicates that the respective quotients in the ratios must be the same, and that the numerator of the $e$ series must be greater than that of the $u$-series by an interval of two times the denominator [eg. $e$-series $(3,8), u$ - series $(3,2)]$. A further surprising phenomenon is that the $j$ indices of both series are identical, and the $i$-indices also except that the $u$-series begins at 3 , causing it to be out of phase with the $i$-indices of the $e$-series. This all goes to show that the indices, even though they may be perceived to have been chosen arbitrarily [1], are meaningful and correct.

Table 4. A comparison of the indices and their ratios of the e-and u-series of $\sqrt{3}$

\begin{tabular}{|c|c|c|c|c|c|}
\hline \multicolumn{3}{|c|}{$e$-series of $\sqrt{3}$} & \multicolumn{3}{|c|}{$u$-series of $\sqrt{3}$} \\
\hline$\underline{i}$ & $i$ & $j / i$ & $i$ & $i$ & $j / i$ \\
\hline 1 & 2 & 2 & 3 & 2 & 0.666666666667 \\
\hline 3 & 8 & 2.666666666667 & 11 & 8 & 0.727272727273 \\
\hline 11 & 30 & 2.727272727273 & 41 & 30 & $0 . \overline{7} 31707317073$ \\
\hline 41 & 112 & $2 . \underline{7} 31707317073$ & 153 & 112 & $0 . \overline{7320} 26143791$ \\
\hline 153 & 418 & 2.732026143791 & 571 & 418 & $0 . \overline{7320} 49036778$ \\
\hline 571 & 1560 & $2 . \overline{7320} 49036778$ & 2131 & 1560 & $0 . \overline{732050680432}$ \\
\hline 2131 & 5822 & $2 . \overline{732050680432}$ & 7953 & 5822 & $0 . \overline{732050} 798441$ \\
\hline 7953 & 21728 & $2 . \overline{732050} 798441$ & 29681 & 21728 & $0 . \overline{732050806914}$ \\
\hline 29681 & 81090 & $2 . \overline{732050806914}$ & 110771 & 81090 & 0.732050807522 \\
\hline 110771 & 302632 & $2 . \overline{732050807522}$ & 413403 & 302632 & $0 . \overline{732050807565}$ \\
\hline 413403 & 1129438 & 2.732050807565 & & & \\
\hline
\end{tabular}

In the triangles of the $u$-series of $\sqrt{3}, 2 e$ is also always 1 less than $h$.

The sides of the triangles $(u, e, h)_{n+1}$ may be defined in terms of $i_{n}$ and $j_{n}$, [(31), (33) and (35) respectively] and in terms of $u_{n}, e_{n}$ and $h_{n}$ [(32), (34) and (36) respectively]:

$$
\begin{gathered}
u_{n+1}=\left(3 i_{n}+j_{n}\right)\left(5 i_{n}+j_{\mathrm{n}}\right) \\
=8 h_{n}-4 e_{n}+7 u_{n} \\
2 e_{n+1}=\left(8 i_{n}+3 j_{n}\right)\left(2 i_{n}+j_{n}\right) \\
\text { and } e_{n+1}=4 h_{n}-e_{n}+4 u_{n} \\
2 h_{n+1}=34 i_{n}^{2}+26 i_{n} j_{n}+5 j_{n}^{2} \\
\text { and } h_{n+1}=9 h_{n}-4 e_{n}+8 u_{n}
\end{gathered}
$$

$h$ may be solved in terms of $h$ only, etc:

$$
\begin{aligned}
h_{n+1} & =15 h_{n}-15 h_{n-1}+h_{n-2} \\
e_{n+1} & =15 e_{n}-15 e_{n-1}+e_{n-2} \\
\text { and } u_{n+1} & =15 u_{n}-15 u_{n-1}+u_{n-2}
\end{aligned}
$$

The general formulae for the three respective sides of the triangles are the same as for the $e$-series. The simple formulae are different, however, in that the members in the respective series differ. The second series of rational numbers that has $\sqrt{3}$ as its limit is therefore the following:

$$
\begin{gathered}
\frac{u_{n+1}}{e_{n+1}} \text { where } u_{1}=15, u_{2}=209, u_{3}=2911, \\
u_{n+1}=15 u_{n}-15 u_{n-1}+u_{n-2}, \\
\text { and } e_{1}=8, e_{2}=120, e_{3}=1680, \\
e_{n+1}=15 e_{n}-15 e_{n-1}+e_{n-2}
\end{gathered}
$$

\section{Series that Have as Their Respective Limits the Roots of All the Uneven Numbers that are Not Perfect Squares}

We have shown that there are series of triangles that have

\begin{tabular}{|c|c|c|c|c|c|c|c|c|c|c|c|c|c|c|c|c|}
\hline hypotenuse & 2 & 3 & 4 & 5 & 6 & 7 & 8 & 9 & 10 & 11 & 12 & 13 & 14 & 15 & 16 & 17 \\
\hline rational leg & 1 & 2 & 3 & 4 & 5 & 6 & 7 & 8 & 9 & 10 & 11 & 12 & 13 & 14 & 15 & 16 \\
\hline irrational leg & $\sqrt{3}$ & $\sqrt{5}$ & $\sqrt{7}$ & 3 & $\sqrt{11}$ & $\sqrt{13}$ & $\sqrt{15}$ & $\sqrt{17}$ & $\sqrt{19}$ & $\sqrt{21}$ & $\sqrt{23}$ & 5 & $\sqrt{27}$ & $\sqrt{29}$ & $\sqrt{31}$ & $\sqrt{33}$ \\
\hline
\end{tabular}
as their limit the $45^{\circ}$ triangle, and the $60^{\circ}$ triangle. These triangles also produce series of rational numbers that have as their limit $\sqrt{2}$ and $\sqrt{3}$, respectively. Which other triangles are there that have two rational sides and one irrational leg? It turns out that the $60^{\circ}$ triangle is the first in a series of irrational right-angled triangles where roots of all the uneven numbers form one leg, and the hypotenuses are consecutive numbers and the other leg, consecutive numbers that are one less than the hypotenuse in each triangle. Table 5 illustrates this with the first triangles of this infinite series.

Table 5. A series of triangles that represent the roots of all the uneven numbers. 
$\sqrt{9}$ and $\sqrt{25}$ are not irrational, and therefore are not a part of the argument, but they do form part of the picture in which the roots of all the uneven numbers are involved. The second triangle in this series forms the limiting triangle of a series of triangles, that will allow us to develop a series of rational numbers that have $\sqrt{5}$ as its limit. Here too, there is an $e$-series and a $u$-series.

The $\boldsymbol{e}$-series of $\sqrt{\mathbf{5}}$ is defined by a subset of triangles (D) as follows:

$$
\begin{gathered}
\mathrm{D}=\left\{(i, j) \mid u=i^{2}+i j, e=j^{2} / 2+i j, h^{2}=u^{2}+e^{2},\right. \\
\text { and }|h-3 u / 2| \leq 1, \text { where }(i+1) / 2 \text { and } j / 2 \in \mathbb{N}\}
\end{gathered}
$$

The mathematical equation to define each member of this series is:

$$
\text { For the series }(i, j)_{n},(i, j)_{n+1}=\left(i_{n}+2 j_{n}, 2 i_{n}+3 j_{n}\right)
$$

Table 6 contains the first ten triangles in this series. $\sqrt{5}$ is included, and the calculated approximation to $\sqrt{5}(2 e / u)$, offered by each respective triangle. The difference between $h$ and $3 u / 2$ is provided, to reveal the eligibility of the triangles for this series. As with $\sqrt{2}$, the difference is alternat-

\begin{tabular}{|c|c|c|c|c|c|c|c|c|}
\hline$n$ & $i$ & $i$ & $u$ & $e$ & $\boldsymbol{h}$ & $3 u / 2$ & Diff & $\begin{array}{l}2 e / u(\sqrt{5}) \\
2.2360679774997897\end{array}$ \\
\hline 1 & 1 & 2 & 3 & 4 & 5 & 4.5 & -0.5 & 2. .66666666666666667 \\
\hline 2 & 5 & 8 & 65 & 72 & 97 & 97.5 & 0.5 & $\underline{2.2} 153846153846154$ \\
\hline 3 & 21 & 34 & 1155 & 1292 & 1733 & 1732.5 & -0.5 & $\underline{2.23} 72294372294372$ \\
\hline 4 & 89 & 144 & 20737 & 23184 & 31105 & 31105.5 & 0.5 & $\underline{2.2360032791628490}$ \\
\hline 5 & 377 & 610 & 372099 & 416020 & 558149 & 558148.5 & -0.5 & $\underline{2.2360715831001965}$ \\
\hline 6 & 1597 & 2584 & 6677057 & 7465176 & 10015585 & 10015585.5 & 0.5 & $\underline{2.2360677765668318}$ \\
\hline 7 & 6765 & 10946 & 119814915 & 133957148 & 179722373 & 179722372.5 & -0.5 & $\underline{2.2360679} 886974005$ \\
\hline 8 & 28657 & 46368 & 2149991425 & 2403763488 & 3224987137 & 3224987137.5 & 0.5 & $\underline{2.2360679768757682}$ \\
\hline 9 & 121393 & 196418 & 38580030723 & 43133785636 & 57870046085 & 57870046084.5 & -0.5 & $\underline{2.2360679775345652}$ \\
\hline 10 & 514229 & 832040 & 692290561601 & 774004377960 & 1038435842401 & 1038435842401.5 & 0.5 & 2.2360679774978517 \\
\hline
\end{tabular}
ing, this time in halves.

Table 6. The e-series of triangles $(i, j)_{n}$ that are used to define a rational series of numbers that have $\sqrt{5}$ as limit.

For the last triangle in the table, the ratio of $j / i$ is determined to be 1.618033988748 .

The sides of the triangles $(u, e, h)_{n+1}$ may be defined in terms of $i_{n}$ and $j_{n}$, [(39), (41) and (43) respectively] and in terms of $u_{n}, e_{n}$ and $h_{n}$ [(40), (42) and (44) respectively]:

$$
\begin{aligned}
u_{n+1} & =\left(3 i_{n}+5 j_{n}\right)\left(i_{n}+2 j_{n}\right) \\
& =12 h_{n}+8 e_{n}-9 u_{n} \\
2 e_{n+1} & =8 i_{n}^{2}+26 i_{n} j_{n}+21 j_{n}^{2} \\
\text { and } e_{n+1} & =12 h_{n}+9 e_{n}-8 u_{n} \\
2 h_{n+1} & =10 i_{n}^{2}+32 i_{n} j_{n}+35 j_{n}^{2} \\
\text { and } h_{n+1} & =17 h_{n}+12 e_{n}-12 u_{n}
\end{aligned}
$$

$h$ may be solved in terms of $h$ only, etc:

$$
\begin{aligned}
h_{n+1} & =17 h_{n}+17 h_{n-1}-h_{n-2} \\
e_{n+1} & =17 e_{n}+17 e_{n-1}-e_{n-2} \\
\text { and } u_{n+1} & =17 u_{n}+17 u_{n-1}-u_{n-2}
\end{aligned}
$$

The first series of rational numbers that has $\sqrt{5}$ as its limit is therefore the following:

$\frac{2 e_{n+1}}{u_{n+1}}$ where $u_{1}=3, u_{2}=65, u_{3}=1155$,

$$
\begin{aligned}
& u_{n+1}=17 u_{n}+17 u_{n-1}-u_{n-2}, \\
\text { and } & e_{1}=4, e_{2}=72, e_{3}=1292, \\
& e_{n+1}=17 e_{n}+17 e_{n-1}-e_{n-2}
\end{aligned}
$$

The $\boldsymbol{u}$-series of $\sqrt{\mathbf{5}}$ is defined by a subset of triangles (E) as follows:

$$
\begin{aligned}
& \mathrm{E}=\left\{(i, j) \mid u=i^{2}+i j, e=j^{2} / 2+i j, h^{2}=u^{2}+e^{2},\right. \\
& \text { and }|h-3 e / 2| \leq 1, \text { where }(i+1) / 2 \text { and } j / 2 \in \mathbb{N}\}
\end{aligned}
$$

The mathematical equation to define each member of this series is:

$$
\text { For the series }(i, j)_{n},(i, j)_{n+1}=\left(3 i_{n}+j_{n}, 4 i_{n}+j_{n}\right)
$$

Table 7 contains the first nineteen triangles in this series. The $\sqrt{5}$ is included, and the calculated approximation to $\sqrt{5}$ $(2 u / e)$, offered by each respective triangle. The difference between $h$ and $3 e / 2$ is provided, to reveal the eligibility of the triangles for this series. The numbers $(n)$ of the triangles are designated $n \mathrm{a}$ and $n \mathrm{~b}$, since the algebra that relates these triangles relates every second triangle. The interval between the indices of $n \mathrm{a}$ and $n \mathrm{~b}$, is equal to the interval between $n \mathrm{~b}$ and $(n+1) \mathrm{a}$, whereas the interval between the 
indices in a series is always geometric. Viewed differently: we thus have two interspersed series of triangles in one. This phenomenon becomes fairly general beyond $\sqrt{5}$. The $u$-series of both $\sqrt{93}$ and $\sqrt{97}$ have thirty interspersed series, respectively. As with $\sqrt{2}$ and the $e$-series of $\sqrt{5}$, this interspersed pair has the difference alternating in sign.

For the last triangle in the table, the ratio of $j / i$ is 1.236067977496. The product of the $j / i$ ratios of the $e$ series and the $u$-series is 2 again, viz. $1.618033988748 \times$ $1.236067977496=2$. This phenomenon is universal, and can be proven as follows:

For an $e$-series and a $u$-series in root $n$, select triangles in the respective series that approximate the limiting triangle well, and are of similar size. Let subscript $e$ represent the $e$-series, and likewise for the $u$-series.

$$
\begin{aligned}
\text { Then } h_{e} & =h_{u}, e_{e}=u_{u}, \text { and } u_{e}=e_{u} \\
h & =i^{2}+e, \text { and } h=j^{2} / 2+u \\
& \text { By definition of } i \text { and } j, \text { ref [1] } \\
\therefore h_{e}=h_{u} & =i_{e}^{2}+e_{e}=j_{u}^{2} / 2+u_{u} \\
\therefore i_{e}^{2} & =j_{u}^{2} / 2 \quad \text { Since } e_{e}=u_{u} \\
\text { and } i_{e} \sqrt{2} & =j_{u}
\end{aligned}
$$

\begin{tabular}{|c|c|c|c|c|c|c|c|c|}
\hline$n$ & $i$ & $i$ & $u$ & $e$ & $\boldsymbol{h}$ & $3 e / 2$ & Diff & $\begin{array}{l}2 u / e(\sqrt{5}) \\
2.2360679774997897\end{array}$ \\
\hline 1a & 1 & 2 & 3 & 4 & 5 & 6 & -1 & 1.5 \\
\hline $1 b$ & 3 & 4 & 21 & 20 & 29 & 30 & -1 & $\underline{2} .1$ \\
\hline $2 \mathrm{a}$ & 5 & 6 & 55 & 48 & 73 & 72 & 1 & $\underline{2.2} 9166666666666667$ \\
\hline $2 b$ & 13 & 16 & 377 & 336 & 505 & 504 & 1 & $\underline{2.2440476190476190}$ \\
\hline $3 \mathrm{a}$ & 21 & 26 & 987 & 884 & 1325 & 1326 & -1 & $\underline{2.23} 30316742081448$ \\
\hline $3 b$ & 55 & 68 & 6765 & 6052 & 9077 & 9078 & -1 & $\underline{2.23} 56245869134171$ \\
\hline $4 \mathrm{a}$ & 89 & 110 & 17711 & 15840 & 23761 & 23760 & 1 & $\underline{2.236} 2373737373737$ \\
\hline $4 b$ & 233 & 288 & 121393 & 108576 & 162865 & 162864 & 1 & $\underline{2.2360} 926908340701$ \\
\hline $5 \mathrm{a}$ & 377 & 466 & 317811 & 284260 & 426389 & 426390 & -1 & $\underline{2.2360585379582073}$ \\
\hline $5 b$ & 987 & 1220 & 2178309 & 1948340 & 2922509 & 2922510 & -1 & $\underline{2.23606} \underline{\underline{2}} 6002853711$ \\
\hline $6 a$ & 1597 & 1974 & 5702887 & 5100816 & 7651225 & 7651224 & 1 & $\underline{2.23606} 85035492360$ \\
\hline $6 b$ & 4181 & 5168 & 39088169 & 34961520 & 52442281 & 52442280 & 1 & $\underline{2.23606} 80542493576$ \\
\hline $7 \mathrm{a}$ & 6765 & 8362 & 102334155 & 91530452 & 137295677 & 137295678 & -1 & $\underline{2.2360679481840645}$ \\
\hline $7 b$ & 17711 & 21892 & 701408733 & 627359044 & 941038565 & 941038566 & -1 & $\underline{2.2360679732226830}$ \\
\hline $8 \mathrm{a}$ & 28657 & 35422 & 1836311903 & 1642447296 & 2463670945 & 2463670944 & 1 & $\underline{2.2360679791334991}$ \\
\hline $8 b$ & 75025 & 92736 & 12586269025 & 11257501248 & 16886251873 & 16886251872 & 1 & $\underline{2.2360679777381447}$ \\
\hline $9 \mathrm{a}$ & 121393 & 150050 & 32951280099 & 29472520900 & 44208781349 & 44208781350 & -1 & $\underline{2.2360679774087462}$ \\
\hline $9 b$ & 317811 & 392836 & 225851433717 & 202007663444 & 303011495165 & 303011495166 & -1 & $\underline{2.2360679774865066}$ \\
\hline $10 \mathrm{a}$ & 514229 & 635622 & 591286729879 & 528862928880 & 793294393321 & 793294393320 & 1 & 2.2360679775048634 \\
\hline
\end{tabular}

Likewise $i_{u} \sqrt{2}=j_{e}$

Table 7. The u-series of triangles $(i, j)_{n}$ that are used to define a rational series of numbers that have $\sqrt{5}$ as limit.

Therefore the product of the $j / i$-ratios of the respective series yields the following:

$$
\begin{aligned}
j_{e} / i_{e} \times j_{u} / i_{u} & =\frac{j_{e} j_{u}}{i_{e} i_{u}} \\
& =\frac{i_{u} \sqrt{2} i_{e} \sqrt{2}}{i_{e} i_{u}} \\
& =2
\end{aligned}
$$

The sides of the triangles $(u, e, h)_{n+1}$ may be defined in terms of $i_{n}$ and $j_{n},[(50),(52)$ and (54) respectively] and in terms of $u_{n}, e_{n}$ and $h_{n}[(51),(53)$ and (55) respectively]:

$$
\begin{aligned}
u_{n+1} & =\left(3 i_{n}+j_{n}\right)\left(7 i_{n}+2 j_{n}\right) \\
& =12 h_{n}-8 e_{n}+9 u_{n} \\
2 e_{n+1} & =\left(10 i_{n}+3 j_{n}\right)\left(4 i_{n}+j_{n}\right) \\
\text { and } e_{n+1} & =12 h_{n}-9 e_{n}+8 u_{n} \\
2 h_{n+1} & =58 i_{n}^{2}+34 i_{n} j_{n}+5 j_{n}^{2} \\
\text { and } h_{n+1} & =17 h_{n}-12 e_{n}+12 u_{n}
\end{aligned}
$$


$h$ may be solved in terms of $h$ only, etc, and the general equation is identical to that of the $e$-series:

$$
\begin{aligned}
h_{n+1} & =17 h_{n}+17 h_{n-1}-h_{n-2} \\
e_{n+1} & =17 e_{n}+17 e_{n-1}-e_{n-2} \\
\text { and } u_{n+1} & =17 u_{n}+17 u_{n-1}-u_{n-2}
\end{aligned}
$$

The second and third series (interspersed series) of rational numbers that have $\sqrt{5}$ as their respective limits are therefore the following:

$$
\begin{aligned}
\frac{2 u_{n+1}}{e_{n+1}} \text { where } & u_{1}=3, u_{2}=55, u_{3}=987, \\
& u_{n+1}=17 u_{n}+17 u_{n-1}-u_{n-2}, \\
\text { and } & e_{1}=4, e_{2}=48, e_{3}=884
\end{aligned}
$$

$$
e_{n+1}=17 e_{n}+17 e_{n-1}-e_{n-2}
$$

and

$$
\begin{aligned}
\frac{2 u_{n+1}}{e_{n+1}} \text { where } & u_{1}=21, u_{2}=377, u_{3}=6765, \\
& u_{n+1}=17 u_{n}+17 u_{n-1}-u_{n-2}, \\
\text { and } & e_{1}=20, e_{2}=336, e_{3}=6052, \\
& e_{n+1}=17 e_{n}+17 e_{n-1}-e_{n-2}
\end{aligned}
$$

An interesting phenomenon, but not unexpected, is the symmetry that there is between the $e$ - and $u$-series with respect to $(u, e, h)_{n+1}$ in terms of $(u, e, h)_{n}$. Table 8 illustrates this phenomenon.

Table 8. A comparison of $(u, e, h)_{n+1}$ in terms of $(u, e, h)_{n}$ of the e- and $u$-series of $\sqrt{3}$ and $\sqrt{5}$.

\begin{tabular}{lllll}
\hline & \multicolumn{5}{c}{$\boldsymbol{e}$-series } & & u-series \\
\hline & $u_{n+1}=4 h_{n}+4 e_{n}-u_{n}$ & $(21)$ & $u_{n+1}=8 h_{n}-4 e_{n}+7 u_{n}$ & $(32)$ \\
$\sqrt{3}$ & $e_{n+1}=8 h_{n}+7 e_{n}-4 u_{n}$ & $(23)$ & $e_{n+1}=4 h_{n}-e_{n}+4 u_{n} \quad(34)$ \\
& $h_{n+1}=9 h_{n}+8 e_{n}-4 u_{n}$ & $(25)$ & $h_{n+1}=9 h_{n}-4 e_{n}+8 u_{n}$ & $(36)$ \\
& $u_{n+1}=12 h_{n}+8 e_{n}-9 u_{n}$ & $(40)$ & $u_{n+1}=12 h_{n}-8 e_{n}+9 u_{n}$ & $(51)$ \\
$\sqrt{5}$ & $e_{n+1}=12 h_{n}+9 e_{n}-8 u_{n}$ & $(42)$ & $e_{n+1}=12 h_{n}-9 e_{n}+8 u_{n}$ & $(53)$ \\
& $h_{n+1}=17 h_{n}+12 e_{n}-12 u_{n}$ & $(44)$ & $h_{n+1}=17 h_{n}-12 e_{n}+12 u_{n}$ & $(55)$ \\
\hline
\end{tabular}

In a similar way series of triangles and therefore rational numbers may be found which have the square roots of all the uneven numbers, that are not perfect squares, as limits, respectively. In table 9 the indices of the first triangle in a series, and the formula for finding the indices for the fol- lowing triangles in that series is provided for the next few series of triangles. Since there are several interspersed subseries per series, the first triangle of each subseries is provided. All subseries in one series have the same formula for finding the next triangle in that subseries.

Table 9. The means to finding the series of several subsets of triangles that follow those discussed.

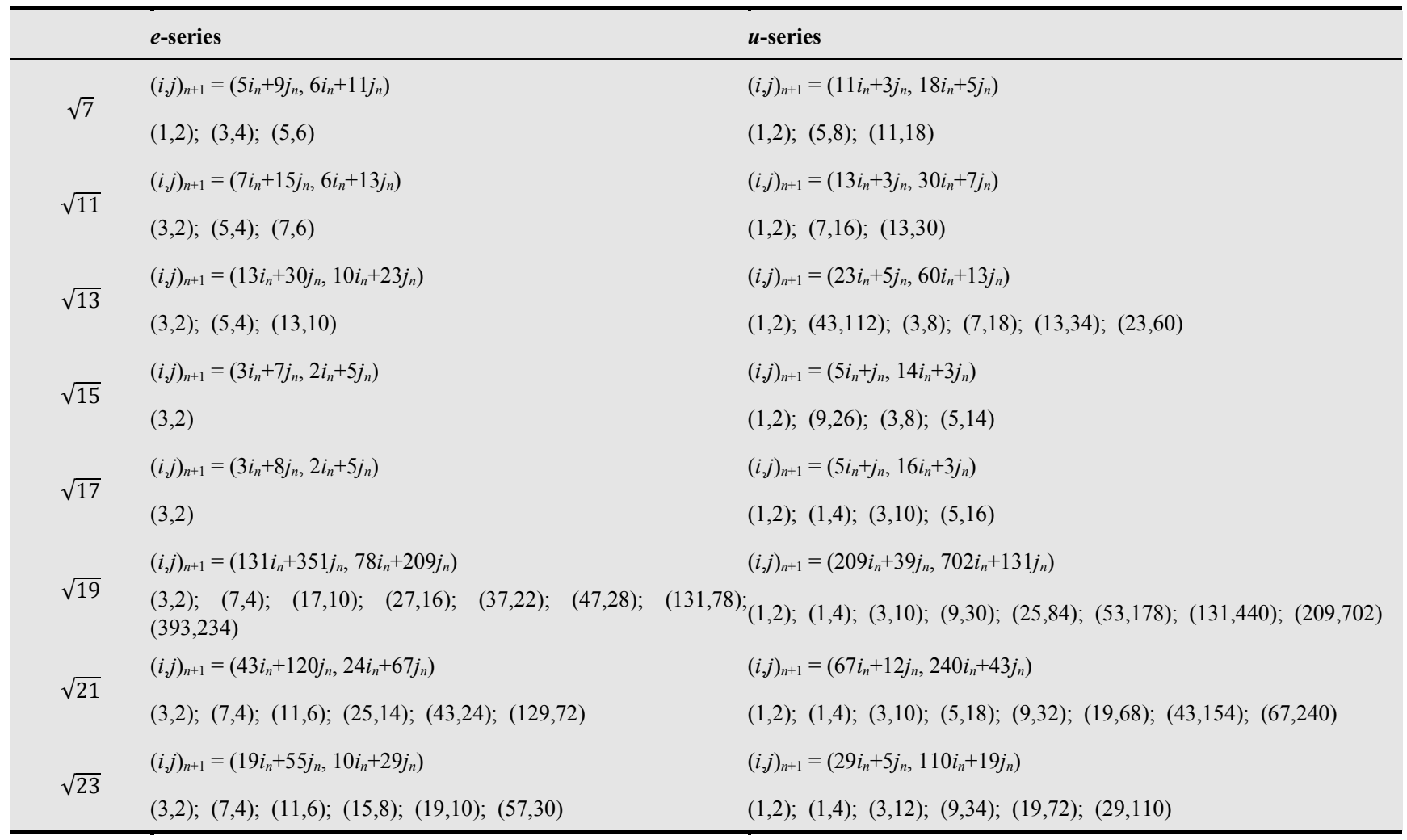

Several observations may be made when studying the table above:
At the $u$-series of $\sqrt{13}$ and $\sqrt{15}$, the second subseries seems to be out of place since it has greater indices than all 
the rest. This is because the respective second subseries do not have a triangle in the first "cycle" of triangles, but starts in the second cycle. If the formula is applied to all the other subseries, replacing the given indices, the second set of indices will fall into place. Stated in another way: the second subseries begins at what should have been the second triangle in the series.

Another observation is that the $i$-index of one of the $e$ subseries is identical to the $i$-index of one of the $u$-subseries throughout the respective series. For example: the fifth subseries of the $e$-series of $\sqrt{21}[(43,24),(4729,2640)$, (520147,290376), ....] has the same $i$-index as the seventh subseries of the $u$-series of $\sqrt{21}[(43,154),(4729,16942)$, $(520147,1863466), \ldots .$.$] .$

Then, as the root increases, going down the table, there are triangles that may be matched, or correlated. In the $e$ series, starting from $\sqrt{11}$, all in the table have triangle $(3,2)$ as first in the first subseries. Triangle $(5,4)$ occurs in both $\sqrt{11}$ and $\sqrt{13}$, etc.

Studying the formulae for propagating the subseries, show interesting systematic correlations. The $i_{n}$-coefficient of both $i$ and $j$ of every such formula in both the series of all the roots, correlate to a starting triangle of one of the subseries being described by the respective formulae. For example: in the $e$-series of $\sqrt{23}$, the respective $i_{n^{-}}$ coefficients of the formula is 19 and 10 . There is a starting triangle $(19,10)$.

Comparing the formulae of the $e$ - and $u$-series of any root has the $i i$-coefficient of the $e$-series equal to the $j j$ coefficient of the $u$-series, and the ji-coefficient of the $e$ - series equal to twice the $i j$-coefficient of the $u$-series. A similar pattern exists for the remaining coefficients.

Testing this technology on a primitive right-angled triangle as limit instead of one with one irrational leg, such as the 3/4/5-triangle, reveals that the $e$-series does not have triangles that fall within 1 when applying the formula: difference between $4 e / 3$ and $h$. The difference diverges. The $u$-series produces a series of triangles where the difference between $4 u / 3$ and $h$ is 0 , when the indices are multiples of $(1,2)$, the indices of the $3 / 4 / 5$-triangle. Therefore there is no convergence, since the series of triangles is the repetition of the 3/4/5-triangle.

A last observation with respect to this family of series of triangles is that when there are several subseries, and a table is created with all the interspersed subseries in ascending order, from a little way down the list of triangles, each triangle that follows may be described as an exact combination of previous triangles. Table 10 illustrates this using the $u$-series of $\sqrt{93}$ as an example. As mentioned earlier in the paper, this series has 30 interspersed subseries, designated $1 \mathrm{a}, \ldots . .1 \mathrm{z}, 1 \mathrm{~A}, 1 \mathrm{~B}, 1 \mathrm{C}, 1 \mathrm{D}$. Thereafter the second member of each subseries follows: $2 \mathrm{a}, 2 \mathrm{~b}$ etc. In the last column, the composition of the indices of that particular triangle is indicated by only the letter of triangles that occur closely above that triangle in the table. Numbers have algebraic meaning such as $2 \mathrm{f}+\mathrm{a}$, for $\mathrm{g}$. This is true for $1 \mathrm{~g}$ and $2 \mathrm{~g}$, etc. It is interesting to note that the composition is always comprised of an uneven number of components. This is because the $i$-indices are uneven, and to remain uneven, the number of components must be uneven.

Table 10. The u-series of $\sqrt{93}$ illustrates the composition of indices from previous triangles.

\begin{tabular}{|c|c|c|c|c|c|c|c|c|}
\hline$n$ & $i$ & $i$ & $u$ & $e$ & $h$ & $47 e / 46$ & Diff & Comp \\
\hline 1a & 1 & 2 & 3 & 4 & 5 & 4.1 & -0.91 & \\
\hline $1 b$ & 1 & 4 & 5 & 12 & 13 & 12.3 & -0.74 & \\
\hline $1 \mathrm{c}$ & 1 & 6 & 7 & 24 & 25 & 24.5 & -0.48 & \\
\hline $1 d$ & 1 & 8 & 9 & 40 & 41 & 40.9 & -0.13 & \\
\hline 1e & 1 & 10 & 11 & 60 & 61 & 61.3 & 0.30 & \\
\hline 1f & 1 & 12 & 13 & 84 & 85 & 85.8 & 0.83 & \\
\hline $1 \mathrm{~g}$ & 3 & 26 & 87 & 416 & 425 & 425.0 & 0.04 & $2 \mathrm{f}+\mathrm{a}$ \\
\hline $1 \mathrm{~h}$ & 5 & 44 & 245 & 1188 & 1213 & 1213.8 & 0.83 & $g+e+d$ \\
\hline 1i & 7 & 60 & 469 & 2220 & 2269 & 2268.3 & -0.74 & $2 g+d$ \\
\hline $1 \mathrm{j}$ & 9 & 78 & 783 & 3744 & 3825 & 3825.4 & 0.39 & $3 g$ \\
\hline $1 \mathrm{k}$ & 13 & 112 & 1625 & 7728 & 7897 & 7896.0 & -1 & $\mathrm{i}+\mathrm{h}+\mathrm{d}$ \\
\hline 11 & 19 & 164 & 3477 & 16564 & 16925 & 16924.1 & -0.91 & $2 \mathrm{i}+\mathrm{h}$ \\
\hline $1 \mathrm{~m}$ & 25 & 216 & 6025 & 28728 & 29353 & 29352.5 & -0.48 & $2 j+i$ \\
\hline 1n & 31 & 268 & 9269 & 44220 & 45181 & 45181.3 & 0.30 & $k+2 j$ \\
\hline 10 & 59 & 510 & 33571 & 160140 & 163621 & 163621.3 & 0.30 & $\mathrm{n}+\mathrm{l}+\mathrm{j}$ \\
\hline $1 p$ & 87 & 752 & 72993 & 348176 & 355745 & 355745.0 & 0.04 & $2 n+m$ \\
\hline $1 \mathrm{q}$ & 115 & 994 & 127535 & 608328 & 621553 & 621552.5 & -0.48 & $\mathrm{o}+\mathrm{n}+\mathrm{m}$ \\
\hline $1 \mathrm{r}$ & 261 & 2256 & 656937 & 3133584 & 3201705 & 3201705.4 & 0.39 & $3 p$ \\
\hline $1 \mathrm{~s}$ & 289 & 2498 & 805443 & 3841924 & 3925445 & 3925444.1 & -0.91 & $q+2 p$ \\
\hline
\end{tabular}




\begin{tabular}{|c|c|c|c|c|c|c|c|c|}
\hline $1 \mathrm{t}$ & 463 & 4002 & 2067295 & 9860928 & 10075297 & 10075296.0 & -1 & $\mathrm{r}+\mathrm{q}+\mathrm{p}$ \\
\hline $1 \mathrm{u}$ & 637 & 5506 & 3913091 & 18665340 & 19071109 & 19071108.3 & -0.74 & $2 r+q$ \\
\hline $1 \mathrm{v}$ & 811 & 7010 & 6342831 & 30255160 & 30912881 & 30912880.9 & -0.13 & $s+2 r$ \\
\hline $1 \mathrm{w}$ & 985 & 8514 & 9356515 & 44630388 & 45600613 & 45600613.8 & 0.83 & $t+2 r$ \\
\hline $1 x$ & 1709 & 14772 & 28166029 & 134351340 & 137272021 & 137272021.3 & 0.30 & $\mathrm{v}+\mathrm{u}+\mathrm{r}$ \\
\hline $1 \mathrm{y}$ & 3331 & 28792 & 107001713 & 510395784 & 521491345 & 521491344.5 & -0.48 & $x+2 v$ \\
\hline $1 \mathrm{z}$ & 4229 & 36554 & 172471307 & 822684324 & 840568765 & 840568765.8 & 0.83 & $2 x+v$ \\
\hline $1 \mathrm{~A}$ & 5851 & 50574 & 330142675 & 1574773212 & 1609007413 & 1609007412.3 & -0.74 & $y+x+v$ \\
\hline 1B & 8371 & 72356 & 675765717 & 3223387444 & 3293461085 & 3293461084.1 & -0.91 & $2 y+x$ \\
\hline $1 \mathrm{C}$ & 10891 & 94138 & 1143870839 & 5456238480 & 5574852361 & 5574852360.0 & -1 & $z+2 y$ \\
\hline 1D & 13411 & 115920 & 1734458041 & 8273326320 & 8453181241 & 8453181240.0 & -1 & $\mathrm{~A}+\mathrm{z}+\mathrm{y}$ \\
\hline $2 \mathrm{a}$ & 15931 & 137702 & 2447527323 & 11674650964 & 11928447725 & 11928447724.1 & -0.91 & $2 \mathrm{~A}+\mathrm{z}$ \\
\hline $2 b$ & 18451 & 159484 & 3283078685 & 15660212412 & 16000651813 & 16000651812.3 & -0.74 & $\mathrm{~B}+\mathrm{A}+\mathrm{z}$ \\
\hline $2 \mathrm{c}$ & 20971 & 181266 & 4241112127 & 20230010664 & 20669793505 & 20669793504.5 & -0.48 & $\mathrm{~B}+2 \mathrm{z}$ \\
\hline $2 d$ & 23491 & 203048 & 5321627649 & 25384045720 & 25935872801 & 25935872800.9 & -0.13 & $\mathrm{C}+\mathrm{B}+\mathrm{z}$ \\
\hline $2 \mathrm{e}$ & 26011 & 224830 & 6524625251 & 31122317580 & 31798889701 & 31798889701.3 & 0.30 & $2 \mathrm{C}+\mathrm{z}$ \\
\hline $2 f$ & 28531 & 246612 & 7850104933 & 37444826244 & 38258844205 & 38258844205.8 & 0.83 & $\mathrm{D}+\mathrm{C}+\mathrm{z}$ \\
\hline $2 \mathrm{~g}$ & 72993 & 630926 & 51381159567 & 245086990256 & 250414968305 & 250414968305.0 & 0.04 & $2 \mathrm{f}+\mathrm{a}$ \\
\hline $2 \mathrm{~h}$ & 122495 & 1058804 & 144703221005 & 690231151188 & 705236176213 & 705236176213.8 & 0.83 & $g+e+d$ \\
\hline $2 \mathrm{i}$ & 169477 & 1464900 & 276989310829 & 1321232862300 & 1349955315829 & 1349955315828.3 & -0.74 & $2 \mathrm{~g}+\mathrm{d}$ \\
\hline $2 \mathrm{j}$ & 218979 & 1892778 & 462430436103 & 2205782912304 & 2253734714745 & 2253734714745.4 & 0.39 & $3 g$ \\
\hline $2 \mathrm{k}$ & 315463 & 2726752 & 959706270545 & 4577777600928 & 4677294505297 & 4677294505296.0 & -1 & $\mathrm{i}+\mathrm{h}+\mathrm{d}$ \\
\hline 21 & 461449 & 3988604 & 2053472506797 & 9795018261604 & 10007953441205 & 10007953441204.1 & -0.91 & $2 \mathrm{i}+\mathrm{h}$ \\
\hline $2 \mathrm{~m}$ & 607435 & 5250456 & 3558288019585 & 16972954844328 & 17341932123553 & 17341932123552.5 & -0.48 & $2 j+i$ \\
\hline $2 \mathrm{n}$ & 753421 & 6512308 & 5474152808909 & 26111587349100 & 26679230552341 & 26679230552341.3 & 0.30 & $k+2 j$ \\
\hline 20 & 1433849 & 12393690 & 19826602967611 & 94572455920860 & 96628378875661 & 96628378875661.3 & 0.30 & $\mathrm{n}+\mathrm{l}+\mathrm{j}$ \\
\hline $2 p$ & 2114277 & 18275072 & 43108731635673 & 205627692705536 & 210097859938265 & 210097859938265.0 & 0.04 & $2 n+m$ \\
\hline $2 q$ & 2794705 & 24156454 & 75320538813095 & 359277297703128 & 367087673740153 & 367087673740152.0 & -0.48 & $\mathrm{o}+\mathrm{n}+\mathrm{m}$ \\
\hline
\end{tabular}

\section{Other Families of Series of Triangles That Have Even and Uneven Roots}

The family of series of triangles are not just limited to all the uneven roots created by a family where the hypotenuse and the rational leg differ by 1 . Another family exists where the hypotenuse and the rational leg differ by 2 , and then one with a difference of 3 etc. Table 11 shows the family where the hypotenuse and the rational leg differ by two. Every second member of the family, though, has all three the sides as multiples of two, and are thus identical to a triangle in the previous family, once having extracted 2 from all the sides. The triangles that offer new limits to a series of triangles, are those that have an uneven hypotenuse and rational leg. The irrational leg is a root of an even number, which is new, since the previous family had only roots of uneven numbers as limits. Not all even numbers are covered in this way, but every eighth number, beginning with $\sqrt{8}$, is new. When we get to $\sqrt{16}$, we have a perfect square, and this triangle $(3 / 4 / 5)$ is of no use as a limit to a series of triangles.

Table 11. A series of triangles that have the hypotenuse 2 greater than the rational leg.

\begin{tabular}{|c|c|c|c|c|c|c|c|c|c|c|c|c|c|c|c|c|}
\hline hypotenuse & 3 & 4 & 5 & 6 & 7 & 8 & 9 & 10 & 11 & 12 & 13 & 14 & 15 & 16 & 17 & 18 \\
\hline rational leg & 1 & 2 & 3 & 4 & 5 & 6 & 7 & 8 & 9 & 10 & 11 & 12 & 13 & 14 & 15 & 16 \\
\hline irrational leg & $\sqrt{8}$ & $\sqrt{12}$ & 4 & $\sqrt{20}$ & $\sqrt{24}$ & $\sqrt{28}$ & $\sqrt{32}$ & 6 & $\sqrt{40}$ & $\sqrt{44}$ & $\sqrt{48}$ & $\sqrt{52}$ & $\sqrt{56}$ & $\sqrt{60}$ & 8 & $\sqrt{68}$ \\
\hline
\end{tabular}

Something else that needs to be mentioned, is that at times the arbitrary limit of 1 , set for qualifying a triangle into a series, is too small, excluding all possible triangles. If the limit is then raised to 5 or 10 , series of triangles may be found. An example is the $e$-series of triangle $1 / \sqrt{8} / 3$. Three interspersed subseries are generated with a difference be- tween $3 u$ and $h$, of 4, 2 and -4 .

There is another family of triangles that will complete the picture, and they are those where the hypotenuse is the irrational number. The $1 / 1 / \sqrt{2}$, where we started this paper, is such a triangle. Table 12 shows the family of triangles, which act as limits to series, where the two rational legs 
differ by 1 . These families differ from the previous, in that

not arithmetic, but geometric. the difference between the numbers being square-rooted is

Table 12. A series of triangles where the hypotenuse is irrational, and the legs differ by 1 .

\begin{tabular}{llllllllllllllllll}
\hline hypotenuse & $\sqrt{5}$ & $\sqrt{13}$ & 5 & & $\sqrt{41}$ & $\sqrt{61}$ & $\sqrt{85}$ & $\sqrt{113}$ & $\sqrt{145}$ & $\sqrt{181}$ & $\sqrt{221}$ & $\sqrt{265}$ & $\sqrt{313}$ & $\sqrt{365}$ & $\sqrt{421}$ \\
rational leg 1 & 2 & 3 & 4 & 5 & 6 & 7 & 8 & 9 & 10 & 11 & 12 & 13 & 14 & 15 & 13 \\
rational leg 2 & 1 & 2 & 3 & 4 & 5 & 6 & 7 & 8 & 9 & 10 & 11 & 12 & 13 & 14 \\
\hline
\end{tabular}

Other series exist here too where the two rational legs differ by 2, 3 etc. These offer alternate series to a root, compared to those we have dealt with already, where all the uneven numbers are accounted for. Some roots have several solutions. For example $\sqrt{65}$ has eight different series, four pairs ( $e$ - and $u$-series of): $\sqrt{65} / 32 / 33 ; \sqrt{65} / 4 / 9 ; 7 / 4 / \sqrt{65}$ and $1 / 8 / \sqrt{65}$.

Table 13 shows all the roots under $\sqrt{100}$, that serve as a limit to a series of triangles and thus rational numbers in this way. Each number has a pair of series excepting $\sqrt{2}$. Where more than one pair is present, the number of pairs are indicated in parentheses. In total there are therefore $107 \frac{1}{2}$ pairs of series. The number of subseries may also be counted, but they are dependent on what the limit of the difference is set to. The limit is 1 most of the time, but at times 5 or 10 .

Table 13. The number of pairs of series that have limits of less than $\sqrt{100}$.

\begin{tabular}{lllllllll}
\hline $2(1 / 2)$ & $13(2)$ & 26 & $35(2)$ & 47 & $57(2)$ & $69(2)$ & 79 & $89(2)$ \\
3 & $15(2)$ & 27 & $37(2)$ & $48(2)$ & $58(2)$ & 71 & $80(2)$ & $91(2)$ \\
$5(2)$ & $17(2)$ & $29(2)$ & $39(2)$ & 50 & $59(2)$ & $72(2)$ & 82 & $93(2)$ \\
7 & 19 & 31 & $40(2)$ & $51(2)$ & $61(2)$ & $73(2)$ & 83 & $95(2)$ \\
8 & $21(2)$ & 32 & $41(2)$ & $53(2)$ & $63(2)$ & 74 & $85(4)$ & $96(2)$ \\
10 & 23 & $33(2)$ & 43 & $55(2)$ & $65(4)$ & $75(2)$ & $87(2)$ & $97(2)$ \\
11 & $24(2)$ & 34 & $45(2)$ & $56(2)$ & 67 & $77(2)$ & $88(2)$ & $99(2)$ \\
\hline
\end{tabular}

\section{Conclusion}

The infinite two dimensional array of Pythagorean triples, developed in the previous paper [1], offers a system of subsets of triangles that approximate right-angled triangles with one side that is irrational. These subsets may be ordered into series, that better and better approximate the limiting triangle with one irrational side. Ratios of the sides of these series, produce series of rational numbers that approach an irrational limit. The way to find these series has been shown, and many other interesting asides with respect to these series of triangles, and thus rational numbers.

\section{References}

[1] MW Bredenkamp, Pure and Applied Mathematics Journal, 2013, 2, 36-41. 